\title{
VLT + UVES spectroscopy of the low-ionization intrinsic absorber in SDSS J001130.56+005550.7
}

\author{
D. Hutsemékers ${ }^{1,2}$, P. B. Hall ${ }^{3,4}$, and J. Brinkmann ${ }^{5}$ \\ ${ }^{1}$ Institut d'Astrophysique, Université de Liège, Allée du 6 Août 17, Bât. B5c, 4000 Liège, Belgium \\ e-mail: hutsemekers@astro.ulg.ac.be \\ 2 European Southern Observatory, Casilla 19001, Santiago 19, Chile \\ 3 Princeton University Observatory, Princeton, NJ 08544, USA \\ 4 Departamento de Astronomía y Astrofísica, Pontificia Universidad Católica de Chile, Casilla 306, Santiago 22, Chile \\ 5 Apache Point Observatory, PO Box 59, Sunspot, NM 88349-0059, USA
}

A\&A, 415, 77-85 (2004), DOI: 10.1051/0004-6361:20031688

Key words. quasars: general - quasars: absorption lines - errata, addenda

Using Eq. (4) and the numerical values quoted in Sect. 4.2 of the Paper, which are all correct, the derived distance $r$ should read $4 \mathrm{kpc} \leq r \leq 16 \mathrm{kpc}$ instead of $9 \mathrm{kpc} \leq r \leq 37 \mathrm{kpc}$, i.e. roughly a factor 2 smaller. Accordingly, the distance quoted in Sect. 5 and in the Abstract should read $r \simeq 10 \mathrm{kpc}$ instead of $r \simeq 20 \mathrm{kpc}$. This error does not affect any of the conclusions of the Paper. We thank John Everett for bringing this error to our attention. 